\title{
Single-Molecule Cloak
}

\section{A nanoparticle can be made partially transparent by placing a molecule in front of it, forming a system that might work as an optical switch.}

\section{By Michael Schirber}

A single molecule can provide partial invisibility, or cloaking, to a nearby gold nanoparticle that is hundreds of times larger [1]. Ordinarily the transmission of light from a laser can be cut in half when obscured by such a nanoparticle, but the molecule's presence causes $10 \%$ more light to be transmitted. With better control of the system, the molecule-nanoparticle combination could potentially become transparent to the laser. The effect-which is based on the interference between the light scattered from the molecule and that scattered from the nanoparticle-could lead to optical switches in which light transmission would be controlled by single molecules.

For more than a decade, researchers have been creating close interactions (coupling) between a gold nanoparticle and a molecule by bringing them close together. Usually the nanoparticle acts as an antenna that funnels light to the

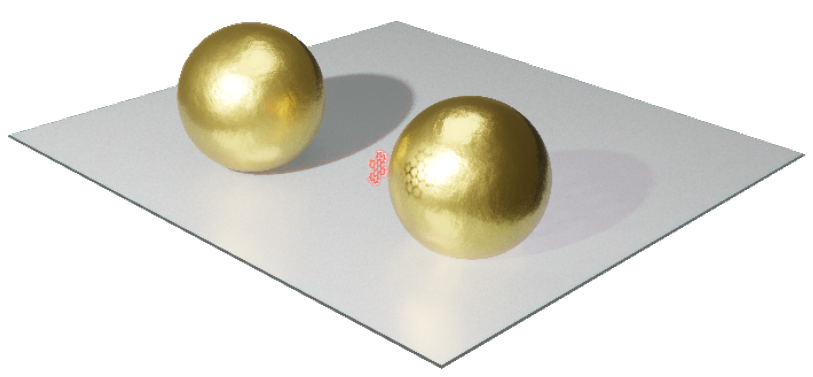

Shadow stealer. The absorption of light by a gold nanoparticle is reduced-and the shadow weakened-when a dye molecule (red) is placed nearby. With experimental improvements, the nanoparticle could become transparent. Credit: J. Zirkelbach/Max Planck Inst. for the Science of Light molecule in order to boost its absorption or emission at a different wavelength. Increasing the emission can make it easier to detect a biomolecule, for example. However, in some cases, researchers want to control the molecule's emission at the same wavelength as the incoming light. If these emission waves are in phase with the light striking the nanoparticle, then an interference effect can lead to a boost in the transmission through the nanoparticle. Previous work predicted that this sort of coherent coupling could make the nanoparticle's shadow disappear [2], but the cloaking effect has been difficult to produce.

Vahid Sandoghdar of the Max Planck Institute for the Science of Light in Germany and his colleagues have succeeded in coherently coupling a dye molecule (dibenzoterrylene) to a 130-nm-wide gold particle. One of the main challenges was placing the molecule and nanoparticle near each other in a solid. The team's strategy was to first fabricate an evenly spaced array of gold nanoparticles on a solid surface and then to surround the particles with a solution containing dye molecules. When the team lowered the temperature, the solution solidified into a transparent crystal with several molecules near each nanoparticle. "We [then] have to fish and see which molecule might be in the right situation next to a gold particle," Sandoghdar says.

For this fishing expedition, the researchers focused a near-infrared laser beam on one of the nanoparticles and recorded the fluorescence signal from the molecules surrounding the targeted nanoparticle. The team was able to pinpoint a molecule with a strong fluorescence emission, suggesting a coherent coupling to its nearby nanoparticle. When the researchers looked at the nanoparticle's transmission, they found that its shadow decreased by $10 \%$ when the laser was tuned to the molecule's absorption 


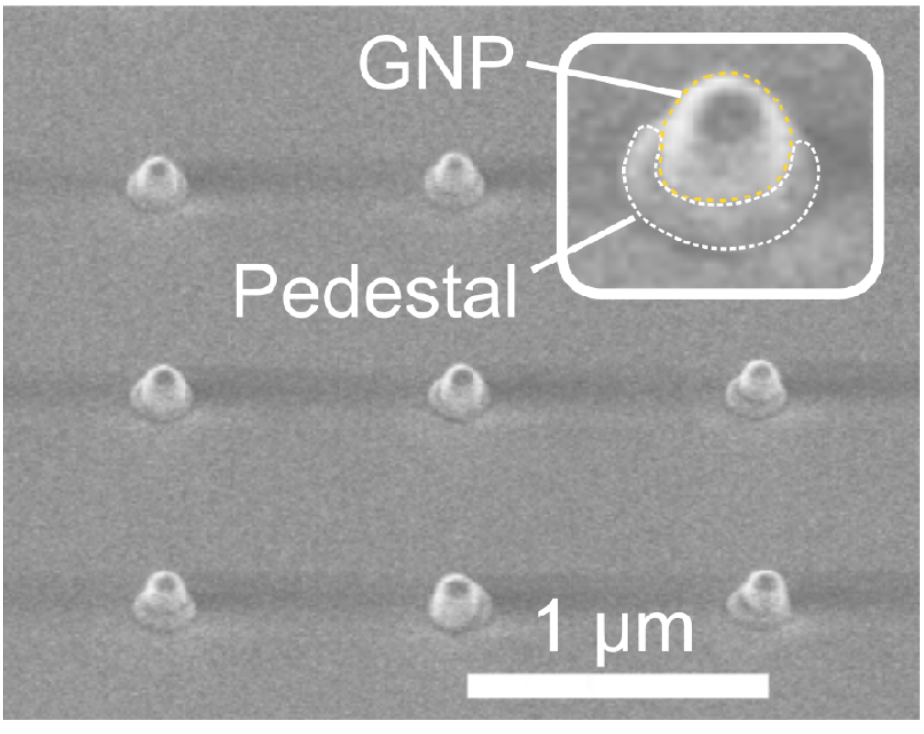

Like peas in a pod. This electron microscope image shows the gold nanoparticles before submersion in the fluid containing dye molecules. The zoomed-in image in the inset shows an example of the glass pedestal that underlies each particle.

Credit: J. Zirkelbach et al. [1]

wavelength. Sandoghdar says that better control of molecule and nanoparticle placement could improve the cloaking.

The molecule-nanoparticle combination could be used as a tiny switch. In such a device, the molecule could toggle between two states of the system: one that blocks light and one that makes the combination transparent. Other systems already can do this-molecule-cavity combinations exhibit near-100\% transparency. However, nanoparticles are at least 10 times smaller than most cavities, which could make them useful as elements in future circuits that use photons rather than electrons.

"I am impressed at how the authors were able to overcome several technical difficulties which prevented this achievement so far," says theorist Salvatore Savasta from the University of Messina in Italy. He imagines that this demonstration could lead to switches and gates that are activated with a single photon [3]. Matthew Pelton, who works on light interactions with nanoparticles at the University of Maryland, Baltimore County, agrees. "The experiments are indeed very challenging, and this group is one of the few in the world that could pull them off at this level," he says. But he says that one of the downsides is that the coherent coupling requires cryogenic temperatures.

Acknowledging this drawback, Sandoghdar says that he and his colleagues are working on enabling the effect at higher temperatures.

Michael Schirber is a Corresponding Editor for Physics based in Lyon, France.

\section{REFERENCES}

1. J. Zirkelbach et al., "Partial cloaking of a gold particle by a single molecule," Phys. Rev. Lett. 125, 103603 (2020).

2. X.-W. Chen et al., "Coherent interaction of light with a metallic structure coupled to a single quantum emitter: From superabsorption to cloaking," Physical Review Letters 110, 153605 (2013).

3. A. Ridolfo et al., "Quantum plasmonics with quantum dot-metal nanoparticle molecules: Influence of the Fano effect on photon statistics," Phys. Rev. Lett. 105, 263601 (2010). 